\title{
Osteonecrose dos Maxilares Induzida por Bisfosfonatos: Relato de Caso Clínico
}

Bisphosphonate-Induced Osteonecrosis of the Jaw: Case Report

\author{
Rogério Jardim Caldas' ${ }^{1}$ José Roberto de Menezes Pontes², Héliton Spíndola Antunes ${ }^{3}$
}

\section{Resumo}

A osteonecrose dos maxilares induzida por bisfosfonatos é a primeira complicação tardia da terapia com bisfosfonatos descrita na literatura científica. Essa é definida como o desenvolvimento de osso necrótico na cavidade oral de um paciente que esteja recebendo tratamento com bisfosfonatos e não tenha recebido radioterapia em região de cabeça e pescoço. Clinicamente, as lesões se caracterizam como ulcerações da mucosa oral, frequentemente muito dolorosas, que expõem o osso subjacente. O objetivo deste trabalho é relatar um caso clínico de osteonecrose induzida por bisfosfonatos de surgimento espontâneo, com difícil resposta a tratamento conservador, alcançando a cura com cirurgia.

Palavras-chave: Osteonecrose; Maxilares; Bisfosfonatos

\footnotetext{
${ }^{1}$ Especialista em Odontologia Oncológica pelo Instituto Nacional de Câncer (INCA); Especialista em Estomatologia pela Universidade Federal do Rio de Janeiro (UFRJ)

${ }^{2}$ Doutorando em Periodontia pela Universidade Estadual do Rio de Janeiro (UERJ); Especialista em Estomatologia pela UERJ; Staff da Seção de Estômato-Odontologia e Prótese do INCA; Professor de Estomatologia e Prevenção do Câncer da UERJ

${ }^{3}$ Doutorando em Oncologia pelo INCA; Mestre em Bioengenharia pela Universidade do Vale do Paraíba (UNIVAP); Especialista em Odontologia Oncológica pelo INCA; Staff do Serviço de Pesquisa Clínica do INCA; Professor do Núcleo Integrado de Estomatologia da UNIGRANRIO Endereço para correspondência: Héliton Spíndola Antunes. Rua André Cavalcanti, 37 - Centro - Rio de Janeiro (RJ), Brasil - CEP: 20231-050. E-mail: hspindola@inca.gov.br
} 


\section{INTRODUÇÃO}

Atualmente, o uso prolongado dos bisfosfonatos está associado com necrose óssea em maxila e mandíbula ${ }^{1,2}$. A osteonecrose dos maxilares (ONM) induzida por bisfosfonatos é a primeira complicação tardia da terapia com bisfosfonatos descrita na literatura científica ${ }^{3,4}$. Essa é definida como o desenvolvimento de osso necrótico na cavidade oral de um paciente que esteja recebendo tratamento com bisfosfonatos e não tenha recebido radioterapia em região de cabeça e pescoço. A ONM pode se desenvolver espontaneamente ou surgir após tratamento dentário (especialmente cirurgias orais). Clinicamente, as lesões se caracterizam como ulceraçōes da mucosa oral e, geralmente, são dolorosas, mas podem se apresentar assintomáticas.

O objetivo deste trabalho é apresentar um caso clínico de ONM de surgimento espontâneo e cura através de cirurgia, bem como descrever as suas características clínicas, histopatológicas e imaginológicas, contribuindo assim para um melhor conhecimento dessa doença.

\section{RELATO DO CASO CLÍNICO}

Paciente de 75 anos, sexo feminino, foi encaminhada, pelo Serviço de Oncologia Clínica do Instituto Nacional de Câncer (INCA), à Seção de Estômato-Odontologia e Prótese, para avaliação de aumento de volume de tecido mole em região de segundo molar superior direito, que se apresentava edêntula. Sua história médica pregressa revelou câncer de mama desde 1986. Na época, a paciente fora submetida à remoção cirúrgica do tumor em mama direita e ao esvaziamento axilar homólogo fora do INCA, sendo encaminhada ao mesmo para tratamento complementar. Realizou-se, então, complementação radioterápica com dose de 46 Gy em sete semanas, em toda a mama direita. Em 2000, quando mestástases ósseas surgiram na coluna cervical e lombar, e na região occipital do crânio, a paciente iniciou tratamento com tamoxifen e pamidronato (Aredia ${ }^{\circledR}$ ). Quatro meses após esse esquema terapêutico, iniciou o uso de zoledronato (Zometa $\left.{ }^{\circledR}\right)$ ao invés do pamidronato.

Vinte e oito meses após início da terapia com zoledronato (outubro/2003), o exame clínico intraoral mostrava lesão granulomatosa localizada em região de segundo molar superior direito, medindo cerca de $0,5 \mathrm{~cm}$ em seu maior diâmetro. Apresentava-se indolor à palpação com presença de secreção purulenta. Ao exame radiográfico, observou-se reabsorção óssea com limites irregulares.

Em 14/11/2003, procedeu-se à remoção cirúrgica da lesão, com prescrição de amoxicilina em associação com clavulonato de potássio por dez dias. O diagnóstico histopatológico indicou processo inflamatório crônico linfoplasmocitário. Nenhuma evidência de malignidade foi detectada.

A partir de então, observava-se úlcera no local da biópsia com exposição óssea que não regredia a tratamento conservador (figura 1), como curetagem (dezembro/2004) e irrigação local pelo profissional e uso diário de gluconato de clorexidina $0,12 \%$ (colutório sem álcool).

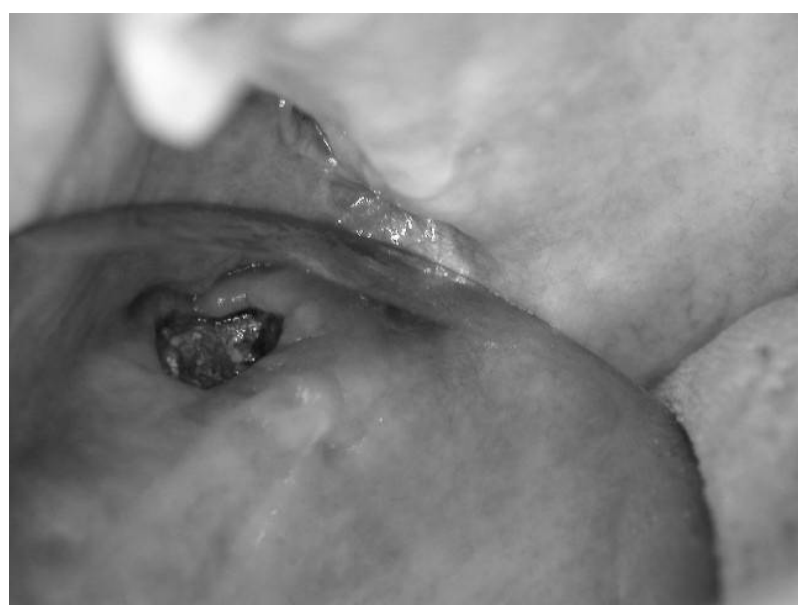

Figura 1. Lesão ulcerada com borda edemaciada e sangrante, apresentando, em área central, exposição de osso necrótico, em região de elemento dentário 17

Dezessete meses após a curetagem sem cicatrização total da úlcera, a tomografia computadorizada de seios da face mostrou fístula bucosinusal, e a cintilografia evidenciou processo inflamatório em maxila à direita. Em maio de 2006, a paciente foi submetida à cirurgia de sequestrectomia e plastia do seio maxilar direito, apresentando ótima cicatrização em rebordo alveolar superior direito (figura 2). A última consulta de controle foi realizada em abril de 2007, quando, ao exame clínico, observou-se mucosa oral íntegra.

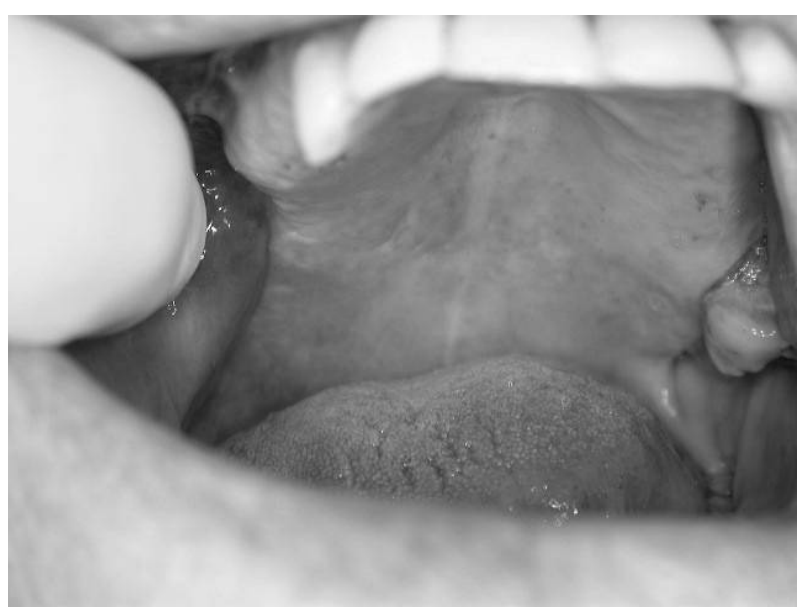

Figura 2. Aspecto clínico de completa cicatrização da região envolvida, um mês após cirurgia 


\section{DISCUSSÃO}

Durie et al. ${ }^{5}$ mostraram o grande impacto do uso de pamidronato e zoledronato sobre o desenvolvimento da ONM. Contudo, o risco cumulativo para tal desenvolvimento é maior em pacientes em tratamento com zoledronato ${ }^{6,7}$. Este alcança $1 \%$ no primeiro ano de tratamento, aumentando para $21 \%$ em três anos ${ }^{6}$. Isso se deve ao fato de que esse fármaco é o bisfosfonato mais potente ${ }^{8}$ o que explica o aumento no número de casos de ONM como o do presente relato.

Apesar de os estudos indicarem ser pertinente a associação da ONM com o tipo de bisfosfonato, a duração da terapia é muito mais significante ${ }^{6,7}$. O estudo de Dimopoulos et al. ${ }^{7}$ mostrou uma ocorrência de ONM significativamente mais cedo entre os pacientes que receberam terapia com zoledronato, cerca de 30 meses após início da terapia. Tal observação pode ser notada neste caso clínico, cujo surgimento da ONM ocorreu após 28 meses do início da terapia com zoledronato.

A osteonecrose é tipicamente progressiva e, com o envolvimento subsequente dos dentes adjacentes, frequentemente leva a uma comprometida higiene oral dos dentes próximos à lesão ${ }^{8,9}$. O prejuízo da higiene oral, em consequência, facilita a infecção local, tornando a necrose óssea mais extensa e aumentando a mobilidade dentária, inclusive chegando à perda. A história odontológica pregressa de muitos casos revelou exodontias recentes, com consequente necrose do osso alveolar e demora na cicatrização do local de extração dentária ${ }^{1,3,4,6,10,11,12,13}$. No presente caso clínico, a paciente não relata ter extraído qualquer de seus dentes em menos de um ano, corroborando o fato de que a ONM pode ocorrer de maneira espontânea. No estudo de Marx et al. ${ }^{13}$, a exposição óssea espontânea envolveu 30 pacientes $(25,2 \%)$.

Ruggiero et al. ${ }^{14}$ relataram 23 pacientes com envolvimento maxilar; 39 com envolvimento mandibular; e um acometimento de maxila e mandíbula. De fato, a ONM pode ser observada tanto na maxila quanto na mandíbula $^{1,3,9,10,12,15,16,17,18,19,20,21}$, sendo a região posterior desta, na área próxima à linha miloioidea, o local mais comum ${ }^{6,11,13}$. Entre os 22 pacientes com ONM, no estudo de Badros et al. ${ }^{11}$, somente dois apresentavam lesões em região anterior e parassinfisária de mandíbula. Merigo et al. ${ }^{22}$ observaram que as recorrências sempre ocorriam na mandíbula. Não é incomum encontrar ulceração traumática da mucosa oposta ao osso necrótico devido ao contato do tecido mole contra a superfície áspera do osso ${ }^{1}$. Injúria ao tecido neural adjacente também pode ocorrer. Há queixas de parestesia dos lábios e da face, sugerindo tal envolvimento ${ }^{1,12,19,21,22}$. Dificuldades de fala, mastigação, trismo e otalgia são queixas de alguns pacientes ${ }^{4,9,22}$. Pode ocorrer fratura patológica ${ }^{23}$.
Acreditava-se que a osteonecrose induzida por bisfosfonatos ocorresse apenas nos maxilares ${ }^{13}$. Entretanto, Polizzotto et al..$^{24}$ relataram o caso de um homem de 64 anos de idade com mieloma múltiplo que recebia terapia com zoledronato e desenvolveu osteonecrose em cavidade oral após extração dentária e no canal auditivo externo do lado esquerdo. Essa região apresentava uma exostose que fora removida há seis meses.

Há autores que discordam da associação causal entre bisfosfonatos e osteonecrose, visto que os pacientes que recebem terapia com esses fármacos frequentemente tomam múltiplas drogas, como antineoplásicos e corticoides $^{25}$. Todavia, neste relato, a paciente fazia uso apenas do tamoxifen, além do bisfosfonato. Também não apresentava outras comorbidades relatadas na literatura, como etilismo, tabagismo ou irradiação na região dos maxilares ${ }^{13,26,27}$. A ação dos bisfosfonatos sobre o metabolismo ósseo os aponta, portanto, como os principais responsáveis pelas lesões.

Uma importante preocupação inicial para todos os pacientes é a possibilidade de metástase óssea na cavidade oral da neoplasia primária; porém a presença de tumor não foi identificada no exame histopatológico. $\mathrm{Na}$ microscopia, os espécimes consistem de osso necrótico com áreas de inflamação crônica representada por infiltrado celular misto ${ }^{1,6,9,10,12,14,15,17, ~ 19, ~ 20, ~ 22, ~} 23,26$. Debris bacteriano pode estar presente ${ }^{6,10,12,14,20,26}$. Hansen et al..$^{28}$ realizaram um estudo de análise histomorfológica da ONM, comparando-a com a osteorradionecrose. Em todos os casos estudados, havia tecido ósseo necrótico. $\mathrm{Na}$ ONM, múltiplas áreas de osso necrótico parcialmente confluentes entremeavam-se por ninhos residuais de osso vital, enquanto, na osteorradionecrose, observavam-se regiōes homogêneas de osso completamente necrótico. Além do infiltrado inflamatório misto, colônias de Actinomyces em contato com osso vital foram um consistente achado histológico na ONM, o que se confirma por vários relatos ${ }^{1,9,10,11,12,20,26 .}$

Exames microbiológicos mostraram a presença de Actinomyces dentro da lesão óssea ${ }^{10,11,12,22}$. Leite et al. ${ }^{9}$ encontraram Streptococci do grupo Viridans. Badros et al. ${ }^{11}$ observaram, também, diversos outros microorganismos como Peptostreptococcus, Streptococcus sp, Eikenella, Prevotella, Porphyromonas e Fusobacterium. Diferentemente, Ruggiero et al. ${ }^{20}$ e Sarathy et al. ${ }^{21}$ observaram crescimento de microbiota oral normal. Houve casos em que as culturas microbianas não mostraram crescimento ${ }^{26}$.

Dependendo do estágio de desenvolvimento da osteonecrose, a avaliação radiográfica não contribui significativamente ${ }^{1,11}$. O envolvimento periodontal progressivo resulta em perda óssea ao redor do dente envolvido $^{1,11,13}$. Contudo, nenhuma alteração radiográfica específica associada com esse processo necrótico foi documentada. Do mesmo modo, o exame radiográfico 
não forneceu grande contribuição para o presente caso clínico. Talvez porque, diferentemente dos pacientes com osteomielite, a reabsorção óssea ativa ou sequestração não ocorre nos estágios iniciais de desenvolvimento da osteonecrose $^{1}$. A cintilografia mostrou áreas de captação de marcadores radioativos na área de osteonecrose em maxila, além de vértebra em coluna vertebral. Essa captação na área da ONM também pode ser observada no estudo de Migliorati et al. ${ }^{1}$ Entre os exames imaginológicos, a tomografia computadorizada permitiu uma melhor caracterização da ONM. A mesma observação foi encontrada em outros relatos clínicos ${ }^{9,12}$.

Não há ainda estratégias de tratamento que alcancem resolução e cura da ONM. A tendência na literatura indica o manejo não cirúrgico dos pacientes com lesões de $\mathrm{ONM}^{1,13,29}$. No entanto, neste caso clínico, a abordagem conservadora não apresentou melhora do quadro, sendo este resolvido com cirurgia. Assim como, no estudo de Abu-id et al..$^{10}$, a maior taxa de cura (88\%) foi encontrada entre os pacientes submetidos a tratamento cirúrgico radical (ressecção marginal e segmental).

\section{CONCLUSÃO}

O caso clínico relatado no presente trabalho apresenta uma importante característica. A cura foi alcançada com tratamento cirúrgico radical, quando, na literatura, há uma tendência à abordagem conservadora da ONM. Além disso, o caso clínico reafirma o surgimento espontâneo da ONM e mostra a importância de se estar atento para essa condição de difícil manejo.

É necessário, ainda, observar que se deve informar a possibilidade de desenvolvimento da ONM aos pacientes que recebem a terapia com bisfosfonatos, visto que a informação disponível referente ao risco de desenvolvimento da ONM é baseada na experiência clínica.

\section{Potencial Conflito de Interesses: Nada a Declarar}

\section{REFERÊNCIAS}

1. Migliorati CA, Schubert MM, Peterson DE, Seneda LM. Bisphosphonate-associated osteonecrosis of mandibular and maxillary bone: an emerging oral complication of supportive cancer therapy. Cancer 2005;104(1):83-93.

2. Ruggiero SL. Bisphosphonate-induced osteonecrosis: OMS perspective. J Oral Maxillofac Surg 2005;63 (8 Suppl):16-7.

3. Marx RE. Pamidronate (aredia) and zoledronate (zometa) induced avascular necrosis of the jaw: a growing epidemic. J Oral Maxillofac Surg 2003;61(9):1115-8.

4. Migliorati CA. Bisphosphonates and oral cavity avascular bone necrosis. J Clin Oncol 2003;21(22):4253-4.
5. Durie BG, Katz M, Crowley J. Osteonecrosis of the jaw and bisphosphonates. N Engl J Med 2005; 353(1):99-102.

6. Bamias A, Kastritis E, Bamia C, Moulopoulos LA, Melakopoulos I, Bozas G, et al. Osteonecrosis of the jaw in cancer after treatment with bisphosphonates: incidence and risk factors. J Clin Oncol 2005;23(34):8580-7.

7. Dimopoulos MA, Kastritis E, Anagnostopoulos A, Melakopoulos I, Gika D, Moulopoulos LA, et al. Osteonecrosis of the jaw in patients with multiple myeloma treated with bisphosphonates: evidence of increased risk after treatment with zoledranic acid. Haematologica 2006;91(7):968-71.

8. Fleisch H. Bisphosphonates: mechanisms of action. Endocr Rev 1998;19(1):80-100.

9. Leite AF, Figueiredo PT, Melo NS, Acevedo AC, Cavalcanti MG, Paula LM, et al. Bisphosphonate-associated osteonecrosis of the jaws. report of a case and literature review. Oral Surg Oral Med Oral Pathol Oral Radiol Endod 2006;102(1):14-21.

10. Abud-id MH, Açil Y, Gottschalk J, Kreusch T. Bisphosphonatassoziierte Osteonekrose des Kiefers. MundKiefer-Gesichtschirurgie 2006;10(2):73-81.

11. Badros A, Weikel D, Salama A, Goloubeva O, Schneider A, Rapoport $\mathrm{A}$, et al. Osteonecrosis of the jaw in multiple myeloma patients: clinical features and risk factors. J Clin Oncol 2006;24(6):945-52.

12. Farrugia MC, Summerlin DJ, Krowiak E, Huntley T, Freeman S, Borrowdale R, et al. Osteonecrosis of the mandible or maxilla associated with the use of new generation bisphosphonates. Laryngoscope 2006;116(1):115-20.

13. Marx RE, Fortin M, Broumand V. Bisphosphonate-induced exposed bone (osteonecrosis/osteopetrosis) of the jaws: risk factors, recognition, prevention and treatment. J Oral Maxillofac Surg 2005;63(11):1567-75.

14. Ruggiero S, Woo V, Mehrotra B, Fantasia J. Osteonecrosis of the jaws associated with the use of the bisphosphonates medications: a report of 60 cases. Oral Surg Oral Med Oral Pathol Oral Radiol Endod 2004;98(2):196-7.

15. Bagan JV, Murillo J, Jimenez Y, Poveda R, Milian MA, Sanchis JM et al. Avascular jaw osteonecrosis in association with cancer chemotherapy: series of 10 cases. J Oral Pathol Med 2005;34(2):120-3.

16. Bagan JV, Jimenez Y, Murillo J, Hernandez S, Poveda R, Sanchis JM et al. Jaw osteonecrosis associated with bisphosphonates: multiple exposed areas and its relationship to teeth extractions. Study of 20 cases. Oral Oncol 2006;42(3):327-9.

17. Cheng A, Mavrokokki A, Carter G, Stein B, Fazzalari NL, Wilson DF et al. The dental implications of bisphosphonates and bone disease. Aust Dent J 2005;50(4S2):S4-S13.

18. Estilo CL, Williams T, Evtimovska E, Tkach L, Halpern JL, Tunick SJ et al. Osteonecrosis of the maxilla and 
madibule: possible drug-induced complication of the bisphosphonate therapy. Oral Surg Oral Med Oral Pathol 2004;97(4):449-50.

19. Melo MD, Obeid G. Osteonecrosis of the jaws in patients wth a history of receiving bisphosphonate therapy: strategies for prevention and early recognition. Journal of American Dental Association 2005;136(12):1675-81.

20. Ruggiero SL, Mehrotra B, Rosenberg TJ, Engroff SL. Osteonecrosis of the jaws associated with the use of the bisphosphonates: a review of 63 cases. J Oral Maxillofac Surg 2004;62(5):527-34.

21. Sarathy AP, Bourgeois SL, Goodell GG. Bisphosphonateassociated osteonecrosis of the jaws and endodontic treatment: two case reports. J Endod 2005;31(10):759-63.

22. Merigo E, Manfredi M, Meleti M, Corradi D, Vescovi P. Jaw bone necrosis without previous dental extractions associated with the use of bisphosphonates (pamidronate and zoledronate): a four-case report. J Oral Pathol Med 2005;34(10):613-17.

23. Schirmer I, Peters H, Reichart PA, Dürkop H. Bisphosphonate und Osteonekrosen im Kieferbereich. Mund-Kiefer-Gesichtschirurgie 2005;9(4):239-45.

24. Polizzotto MN, Cousins V, Sschwarer AP. Bisphosphonateassociated osteonecrosis of the auditory canal. Br J Haematol 2006;132(1):114.
25. Tarassoff P, Csermak K. Avascular necrosis of the jaws: risk factors in metastatic cancer patients. J Oral Maxillofac Surg 2003;61(10):1238-9.

26. Lenz JH, Steiner-krammer B, Schimidt W, Fietkau R, Mueller PC, Gundlach KK. Does avascular necrosis of the jaws in cancer patients only occur following treatment with bisphosphonates? J Craniomaxillofac Surg 2005;33(6):395-403.

27. Soriano YJ, Bagan JV. Los bisfosfonatos, nueva causa de osteonecrosis maxilar por fármacos: situación actual. Med Oral Patol Oral Cir Bucal 2005;10(Suppl 12):E88-E91.

28. Hansen T, Kunkel M, Weber A, Kirkpatrick CJ. Osteonecrosis of the jaws in patients treated with bisphosphonates - histomorphologic analysis in comparison with infected osteoradionecrosis. J Oral Pathol Med 2006;35 (3):155-60.

29. Damato K, Gralow J, Hoff A, Huryn J, Marx R, Ruggiero $S$, et al. Expert panel recommendation for the prevention, diagnosis and treatment of osteonecrosis of the jaw. dockets management, US food and drug administration. 2005 March [citado em: 2006 jul] Disponível em: http:// www.fda.gov/ohrms/dockets/ac/05/briefing/20054095B2_02_12-Novartis-Zometa-App-11.pdf

\section{Abstract}

The jaws osteonecrosis induced by bisphosphonates is the first late complication of therapy with bisphosphonates described in scientific literature. This is defined as the development of necrotic bone in the oral cavity of a patient who is receiving treatment with bisphosphonates and did not receive radiotherapy in the head or neck region. Clinically, the lesions are characterized as ulceration of the oral mucosa, which is often very painful, and exposes the underlying bone. This paper reports a case of osteonecrosis induced by bisphosphonates of spontaneous surging, difficult to respond to conservative treatment, then achieving a cure with surgery.

Key words: Osteonecrosis; Jaw; Bisphosphonates 